\title{
Factores determinantes para la creación de valor en las universidades chilenas
}

\author{
Sergio Zuniga-Jara ${ }^{{ }^{\star}}$, Oscar Sjoberg-Tapia ${ }^{2}$, Jacobo Rojas-Guerra ${ }^{1}$ y Tamara Valenzuela-Cortes ${ }^{1}$ \\ (1) Escuela de Ciencias Empresariales, Universidad Católica del Norte, Coquimbo, Chile. \\ (correo-e: sz@ucn.cl; jrg030@alumnos.ucn.cl; tvalenzuelacortes@gmail.com) \\ (2) Facultad de Cs. Empresariales, Universidad Arturo Prat, Av. Arturo Prat 2120, Iquique, Chile \\ (correo-e: osjoberg@unap.cl) \\ * Autor a quien debe ser dirigida la correspondencia.
}

Recibido Dic. 15, 2019; Aceptado -Feb. 11, 2020; Versión final Abr. 28, 2020, Publicado Dic. 2020

\begin{abstract}
Resumen
En este estudio se realizaron estimaciones de la creación de valor de 55 universidades chilenas y se discuten los factores más influyentes. Se incluyó las universidades estatales, las privadas de la red G-9 y las privadas nacidas después de la reforma universitaria de 1981. El trabajo se enmarca en la creciente preocupación por la calidad de la educación superior chilena y la eficiencia en el gasto público, que llevó a la promulgación de la llamada ley de educación superior ( $N^{\circ}$ 21.091). Se usó la fórmula tradicional de Stern con adaptaciones menores. Como resultado se obtuvo que todos los grupos de universidades chilenas destruyeron valor. Además, el mejor predictor de desempeño fue el tamaño de las universidades, aunque con un signo inverso. Se concluye que las universidades más grandes son las que estadísticamente tienen la mayor destrucción de valor.
\end{abstract}

Palabras clave: valor económico agregado; gestión universitaria; educación superior; desempeño

\section{Determining factors for value creation in Chilean universities}

\begin{abstract}
The purpose of the present study is to make value creation estimates and discuss their most influential factors in 55 Chilean universities. This study includes Chilean public universities, private universities (G-9 network), and private universities created after the 1981 Chilean university reform. The framework is the higher education law (No. 21,091) enacted recently in Chile, which raised concerns about Chilean higher education quality and public spending efficiency. The traditional Stern formula is applied with minor adaptations. The results show that all types of Chilean universities lost value. In addition, the best predictor of performance is university size, but with an inverse sign. It is concluded that the largest universities are, statistically, the universities that have the most destruction of value.
\end{abstract}

Keywords: economic value; university management; higher education; performance 


\section{INTRODUCCIÓN}

La educación superior en su naturaleza tiene como objetivo contribuir al desarrollo de las personas en diferentes expresiones: sociales, culturales, tecnológicas, científicas y económicas. La UNESCO (1991) considera que la función social de la educación superior se guía fundamentalmente por la búsqueda de la excelencia en la enseñanza, la formación, la investigación y el desempeño institucional. El grado en que estas organizaciones logren estos objetivos es una medida del desempeño de las mismas, lo que es importante en el desarrollo y mejoramiento de la formación universitaria. Este desempeño es especialmente importante, ya que en la mayoría de los países los gobiernos aportan cantidades significativas de dinero al sistema. Entonces es necesario buscar el mayor beneficio social posible de tales recursos. En el caso de las corporaciones privadas, el desempeño se mide simplemente a través de la creación de valor en un período. El valor se mide en moneda corriente, valorando todos los costos e ingresos, sean explícitos e implícitos. A diferencia de esto, las instituciones de educación superior son más complejas ya que persiguen varios objetivos simultáneamente. Además, muchos de los principales bienes y servicios que generan no son necesariamente mostradas en los estados financieros. El desafío es entonces definir un criterio que permita una medida razonable de valoración de cada uno de estos productos.

Para Rescher (1969 y 1982) el criterio de búsqueda de creación de valor es aplicable a cualquier organización social, aunque debe discutirse aquí el significado de 'valor'. Para Baran (1991) el valor se refiere a algo que (por cualquier razón) se ha hecho hincapié y es deseable y contundente para el que se evalúa. El concepto de 'contundencia' se destaca refiriéndose a la internalización de valores relevantes para la acción (fuerzas psicológicas). Para Meynhardt (2009), con la aparición de la 'Nueva Gestión Pública' (Moore, 1995), el concepto de Valor Público (del Estado) reflejaría la opinión de que ese sector no puede reducirse a análisis de costo-beneficio individual, y que se entiende en un sentido amplio, no solo las empresas estatales, sino también aquellas que proporcionan bienes públicos, como la educación superior. Los rankings internacionales de calidad analizan el desempeño de las universidades, aunque parcialmente. Por ejemplo, el SClmago Institutions Rankings (Bornmann, et al. 2012) se enfoca en medir la productividad científica, la innovación y el vínculo con la sociedad, centrándose en los productos finales generados, sin considerar la eficiencia en el uso de los recursos utilizados (Aguillo, et al. 2010). Si solo se miden productos finales, se tenderá a favorecer a las instituciones más grandes. Un elemento que allí no se incluye son costos de producción y el costo del capital en infraestructura, edificios, laboratorios, entre otros.

El enfoque tradicionalmente usado en la literatura científica para analizar el desempeño de las universidades se basa en el análisis comparativo de las entradas (inputs) respecto de las salidas generadas (outputs). Se trata de varios métodos relacionados entre sí, siendo el método base el "Data Envelopment Analysis (DEA, o Análisis Envolvente de Datos). Esta es una técnica de programación lineal desarrollada por originalmente por Charnes, Cooper y Rhodes (1978). Varios estudios han hecho estimaciones de desempeño en universidades a través del mundo usando el enfoque del DEA. Por ejemplo, Daraio et al. (2015) analizó 400 universidades en 16 países europeos en el período: 2005-2009 usando el "Directional distance approach". Porter y Toutkoushian (2006) analizó 203 universidades nacionales y 143 colegios nacionales de arte liberal de los Estados Unidos en el período 1996-1997, utilizando mínimos cuadrados de tres etapas para la estimación. En Chile, Ramírez y Alfaro (2013) analizaron la eficiencia de las universidades pertenecientes al Consejo de Rectores de las Universidades Chilenas (se excluyen todas las universidades privadas que no pertenecen a la llamada Red G-9). Usando la técnica de DEA consideraron como input los gastos operativos, y dos variables de output (publicaciones ISI y número de estudiantes matriculados). Como resultado sólo tres de las 25 universidades fueron eficientes en investigación y docencia (U. de La Serena, U Católica de la Santísima Concepción, y la U. Austral de Chile). Tampoco encontraron diferencias significativas de eficiencia entre las universidades estatales y las no estatales. El trabajo de Shamohammadi y Oh (2019) ofrece una buena revisión de los estudios que han usado estas técnicas en universidades.

A diferencia del enfoque del Data Envelopment Analysis, comentado hasta aquí, en este estudio se usa uno diferente. Se trata del concepto de Valor Económico Agregado (EVA, de la empresa de consultoría fundada en Nueva York en 1982 por Joel M. Stern y G. Bennett Stewart III), el que proporciona una base para esto. El EVA es el excedente disponible que una organización crea después de que es capaz de pagar los gastos de un periodo, y además de satisfacer alguna rentabilidad mínima por el uso del capital. Después de realizar las mediciones, si el EVA tiene un valor positivo, la organización está generando valor; y si tiene un valor negativo, está destruyendo el valor (Stern, Stewart y Chew, 1995). Más específicamente, el EVA se calcula como la diferencia entre el beneficio operativo neto después de impuestos (NOPAT) y el costo de oportunidad del capital invertido. Este costo de oportunidad se determina multiplicando el coste medio ponderado de capital (WACC) y la cantidad de capital empleado. La fórmula es EVA = NOPAT - WACC * Capital (Stern y Shierly, 2001). Una de las principales contribuciones del EVA, en comparación con otras métricas de desempeño habituales, es que incluye todos los costos incurridos para obtener los bienes o servicios finales producidos por cualquier organización social. 
El EVA es consistente con la idea que, para evaluar el desempeño de cualquier institución, no sólo cuenta el valor de los productos ofrecidos, sino también todos los gastos incurridos. Es por eso que el EVA está más alineado con la creación de valor (Grant, 2002). Muchas empresas lo han adoptado como parte de un sistema de gestión y compensación de incentivos. Sharma y Kumar (2010) realizaron una revisión de la literatura, evidenciando la aceptación que esta técnica ha estado obteniendo como una herramienta de medición del rendimiento. Otra ventaja del EVA es su aplicabilidad, ya que puede usarse para medir el desempeño de cualquier tipo de organización (Stern et al., 1995).

Sin embargo, hasta ahora, muy pocas investigaciones de EVA están dirigidas a organizaciones sin fines de lucro. Porter y Kramer (2019) indican que la creación de valor compartido supone una verdadera revolución al fomentar que las Universidades hagan uso de sus habilidades, recursos, capacidades y competencias para liderar el progreso social junto a la comunidad en que está inserta. Rompho (2009) diseñó un modelo de medición del desempeño para una universidad pública, e integró el EVA y el Balance Scorecard. Recientemente, Zuniga-Jara, et al., (2018) proponen un marco novedoso para mejorar la gestión universitaria basada en el EVA, haciendo mediciones en varias facultades de una universidad tradicional chilena. El artículo muestra que el enfoque de análisis EVA es factible de implementar en cualquier universidad. Se identifican las facultades que presentaron las mejores y peores actuaciones, la identificación de las principales causas específicas en cada caso. La evidencia internacional parece indicar que las universidades privadas han logrado una evolución más rápida de lo esperado (Tang, 2012). Existe alguna evidencia de diferencias en el foco de los gastos entre ambos tipos de universidades, lo que puede explicar en gran medida tales diferencias (Wilkinson y Yussof, 2005).

Es evidente que coexisten instituciones muy diferentes dentro del sistema universitario, lo que demuestra que la diversidad de enfoques institucionales y académicos y las diferentes formas de trabajo dificultan la comparación de las universidades, incluida la comparación entre grupos. A pesar de ello, esta investigación busca contribuir a la literatura existente del método EVA, y específicamente al caso de organizaciones sin fines de lucro como las universidades. Con ello se busca responder la pregunta sobre la gestión de los recursos invertidos en educación y, sobre todo, se logra medir el desempeño en términos operativos.

Hasta 1981 el sistema universitario chileno estaba compuesto por solo ocho universidades, la mayoría privadas, pero con un importante presupuesto del Estado. A partir de ese año se llevó a cabo una liberalización importante bajo la exigencia de que las nuevas universidades deben ser instituciones sin fines de lucro. Esta liberalización condujo a la creación de 50 universidades en solo una década, y hoy día existen 58 universidades. El resultado de las nuevas universidades privadas ha sido mixto (Cruz-Coke, 2004). Por un lado, algunas han perdido el reconocimiento oficial y se han cerrado o están en proceso de cierre. Sin embargo, otras han sido tremendamente exitosas, pudiendo posicionarse en pocos años en los niveles más altos de los rankings de calidad nacionales e internacionales.

Actualmente la calidad de la educación superior (terciaria) chilena ha sido foco de mucha atención, la que está fuertemente vinculada a la eficiencia del gasto público. Como resultado, la Ley 21.091 de Educación Superior entró en vigor en Chile en el año 2018. Esta ley fortalece el sistema nacional de aseguramiento de la calidad de la educación, y determina los requisitos que las universidades deben cumplir para acceder a la financiación estatal. En 2015 el Estado de Chile entregó US \$ 2.297 millones a la educación superior, lo que equivale al 4,2\% del gasto del Gobierno Central de Chile (Contraloría General de la República, CGR, 2017). La mayoría de estos fondos estatales fueron asignados las universidades estatales (36\%) y universidades privadas G-9 (51\%). Las universidades privadas no G-9 recibieron un $12 \%$, principalmente de modo indirecto, como ayudas a estudiantes de bajos ingresos.

En lo que sigue, el objetivo es analizar el rendimiento global de todas las 55 universidades chilenas, públicas y privadas. Se busca medir la creación de valor económico de estas universidades en el año 2017, que es el año más reciente con información disponible. Por esta vía se busca evaluar su desempeño, medido por la creación de valor para la sociedad, de acuerdo con los criterios establecidos en la metodología.

\section{METODOLOGÍA}

Las medidas tradicionales de evaluación del desempeño de las organizaciones incluyen el cálculo de indicadores como el retorno sobre el patrimonio (ROE= Ingreso neto / Patrimonio), y el retorno sobre los activos (ROA= Ingreso neto / Activos). Dichas medidas tradicionales han sido criticadas por no incluir el costo de los recursos de capital de la organización (Hasani y Fathi, 2012). Por otro lado, el análisis DEA (Data Envelopment Analysis) ha sido ampliamente utilizado para esto, sin embargo, sólo incluye los costos (inputs) explícitos, sin considerar el costo de uso del capital. El criterio del Valor Económico Agregado (EVA) es una medida propuesta para superar estas limitaciones (AI Mamun, Entebang, y Mansor, 2012), sobre el cual también se han hecho propuestas de su aplicabilidad en el sector público (Díaz, W. (2009). 
El EVA se puede calcular como el beneficio operativo neto después de impuestos menos un cargo por el costo de oportunidad del capital invertido. El principio subyacente es determinar si la compañía está obteniendo una tasa de rendimiento más alta que el costo de los fondos invertidos. Si se logra esto, implica que la administración está agregando valor. La fórmula básica para EVA es:

$E V A=$ NOPAT - CAPITAL ${ }^{*} C C P P$

Donde NOPAT = Beneficio operativo neto después de impuestos, CCPP es el costo de capital, CAPITAL es el valor del capital invertido en la organización (total de activos). NOPAT (Net Operating Profit After Tax) representa el beneficio contable que se ha generado debido a las operaciones corrientes. Esto excluye los gastos relativos al financiamiento ya que se busca medir el desempeño de la operación (los intereses de la deuda no son un gasto operacional). Tampoco es operacional la venta de un activo fijo, por ejemplo. En resumen, se incluye el gasto de depreciación, y se excluyen ingresos y gastos no relacionados con la actividad principal de la universidad.

A veces se establece que NOPAT $=\mathrm{EBIT}^{*}(1-\mathrm{Tax})$, donde EBIT (Earnings Before Interest and Taxes) es el beneficio antes de intereses e impuestos. A partir de la Eq. 1, el concepto básico de EVA es que el rendimiento debe medirse en términos del valor agregado durante el período posterior a incluir todos los costos, incluido el costo de capital, dado por "CAPITAL*CCPP". En este estudio, en lugar de la CPPC, se usa aquí la tasa de descuento social (SDR). El término "CAPITAL*SDR" corresponde al "Costo Social del Capital", y es una estimación del costo incurrido por la sociedad por tener cantidades de dinero asignadas a una inversión social, en lugar de asignarlas a cualquier alternativa con el mayor impacto social.

Aunque se mantiene la lógica detrás del EVA tradicional, fue adaptado a un contexto más general, que es aplicable al caso de las universidades (Zuniga-Jara, et al., 2018). Aunque se puede incluir una serie de ajustes adicionales para mejorar la forma en que EVA mide la creación de valor en estas instituciones, en este estudio se busca un enfoque simplificado y directo.

\section{RESULTADOS Y DISCUSIÓN}

La base de datos fue construida en base a los estados financieros de cada universidad para el año 2017. La principal fuente de información es el Servicio de Información de la Educación Superior (SIES). Los pesos chilenos se convirtieron a dólares estadounidenses con un tipo de cambio de $\$ 700$. Para la TSD se ha utilizado la tasa utilizada por el gobierno chileno para proyectos públicos, que es del $6 \%$ (www.ministeriodesarrollosocial.gob.cl).

Para el análisis las 55 universidades se clasificaron en tres grupos principales: (1) Universidades Privadas "nuevas", creadas después de la reforma de 1981 (no pertenecen a la Red G-9 y no reciben financiación estatal directa). Se separó este grupo en dos: aquellas que tienen cuatro o más años de acreditación (Grupo A), y aquellas que tienen menos de cuatro años de acreditación (Grupo B). (2) Universidades privadas tradicionales (universidades privadas organizadas en la llamada Red G-9). (3) Universidades de propiedad estatal. En lo que sigue, sólo se consideran universidades con información financiera completa en el período 2017. Las universidades en proceso de cierre fueron excluidas. Además, se eliminaron tres universidades por no tener información completa en la base de datos: U. Iberoamericana de Ciencias y Tecnología (UNICIT); U. del Mar; y la U. de Arte y Ciencias Sociales (ARCIS). Las estatales Universidad de O'Higgins y Universidad de Aysén no se incluyeron por ser de reciente creación.

La Tabla 1 muestra que la mayor parte de los estudiantes universitarios chilenos se encuentran matriculados en las nuevas universidades privadas (Grupo A y B). Además, si bien el tamaño promedio de una universidad chilena es de alrededor de 13.000 estudiantes, estas nuevas universidades no son mucho más pequeñas que las tradicionales. La misma tabla revela que todas las universidades reportaron resultados operacionales positivos. Estos son los resultados que están relacionados directamente con la actividad principal de las universidades. Entre los componentes no operacionales se puede mencionar los relacionados con los costos financieros. En cualquier caso, la Tabla muestra que desde este punto de vista el desempeño de las universidades del G-9 es bastante superior al resto, ya sea en promedio o globalmente.

La Tabla 2 muestra los componentes para el cálculo del EVA. La tabla muestra que, para todo el sistema, el costo del capital supera en tres veces los resultados operacionales. Como consecuencia se tiene una destrucción neta de valor a nivel global de US\$371,555.000. En efecto, los cuatro grupos de universidades no logran generar suficientes ganancias para compensar sus costos de capital. Los niveles de costo por uso de capital (principalmente infraestructura) son elevados para las universidades estatales, superando a los demás grupos. En adición, los resultados operacionales de este grupo deberían crecer más de siete veces para compensar el costo del capital. Esto es lo que principalmente explica el hecho que sean las universidades estatales las que destruyen más valor. 
Tabla 1. Datos de las universidades, por grupo de propietarios, año 2017. Monedas en miles de dólares estadounidenses. Datos de los estados financieros auditados 2017 del SIES.

\begin{tabular}{|l|l|l|l|l|l|l|}
\hline Grupo & $\begin{array}{l}\text { Número de } \\
\text { Universidades }\end{array}$ & $\begin{array}{l}\text { Matriculas } \\
\text { Totales }\end{array}$ & $\begin{array}{l}\% \\
\text { Matricula }\end{array}$ & $\begin{array}{l}\text { Matriculas } \\
\text { Totales } \\
\text { Promedio }\end{array}$ & $\begin{array}{l}\text { Resultado } \\
\text { Operacional } \\
\text { Promedio }\end{array}$ & $\begin{array}{l}\text { Resultado } \\
\text { No Operacional } \\
\text { Total }\end{array}$ \\
\hline Privadas A & 15 & 238,667 & $32.7 \%$ & 15,911 & 3,510 & 52,643 \\
\hline Privadas B & 15 & 140,507 & $19.3 \%$ & 9,367 & 684 & 10,255 \\
\hline Privadas G-9 & 9 & 155,752 & $21.4 \%$ & 17,306 & 11,197 & 100,774 \\
\hline Estatales & 16 & 194,313 & $26.6 \%$ & 12,145 & 1,629 & 26,057 \\
\hline Total & 55 & 729,239 & $100.0 \%$ & 13,259 & 3,450 & 189,729 \\
\hline
\end{tabular}

Tabla 2. Componentes de EVA, año 2017. Cantidades en miles de dólares estadounidenses.

\begin{tabular}{|l|l|l|l|l|l|}
\hline Grupo & $\begin{array}{l}\text { Resultado } \\
\text { Operacional Total (1) }\end{array}$ & $\begin{array}{l}\text { Costo del } \\
\text { Capital Total (2) }\end{array}$ & $(2) /(1)$ & $\begin{array}{l}\text { EVA } \\
\text { Total }\end{array}$ & $\begin{array}{l}\text { EVA } \\
\text { Promedio }\end{array}$ \\
\hline Privadas A & 52,643 & $(149,541)$ & 2.8 & $(96,898)$ & $(6,460)$ \\
\hline Privadas B & 10,255 & $(43,649)$ & 4.3 & $(33,394)$ & $(2,226)$ \\
\hline Privadas G-9 & 100,774 & $(174,346)$ & 1.7 & $(73,572)$ & $(8,175)$ \\
\hline Estatales & 26,057 & $(193,748)$ & 7.4 & $(167,690)$ & $(10,481)$ \\
\hline Total & 189,729 & $(561,284)$ & 3.0 & $(371,555)$ & $(6,756)$ \\
\hline
\end{tabular}

La Figura 1 muestra los resultados del creación-destrucción de valor EVA a nivel de cada universidad. Las universidades están individualizadas en la Tabla 3, donde se entregan estimaciones más detalladas sobre estos resultados. Aquí, cuatro universidades se destacan por presentar resultados especialmente negativos en comparación con las otras universidades de su grupo: U. de Los Andes, U. de Las Américas, Pontificia U. Católica de Chile, y U. de Chile. Justamente estas dos últimas universidades son precisamente las que tienen el mejor desempeño en el ranking internacional de calidad. Otro aspecto que es posible observar es que todas las universidades estatales presentan EVA negativos, contrario a los otros grupos, en que se tienen resultados mixtos.

Universidades Privadas A

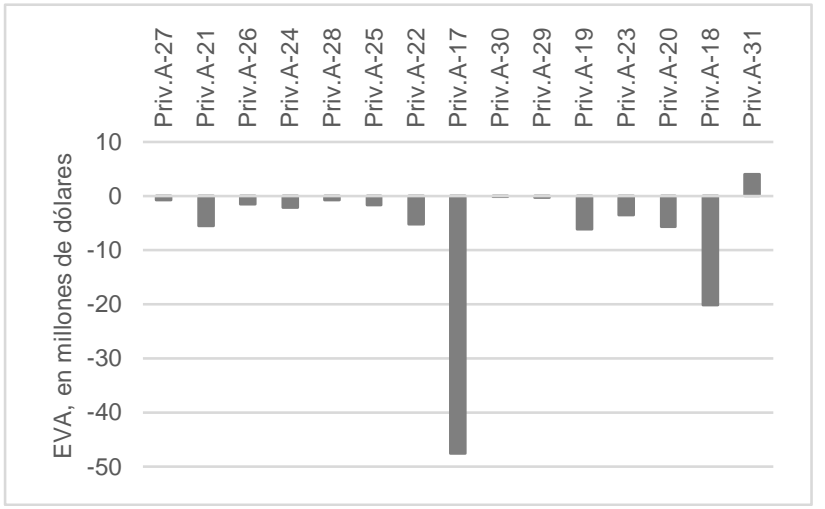

Universidades Privadas G-9

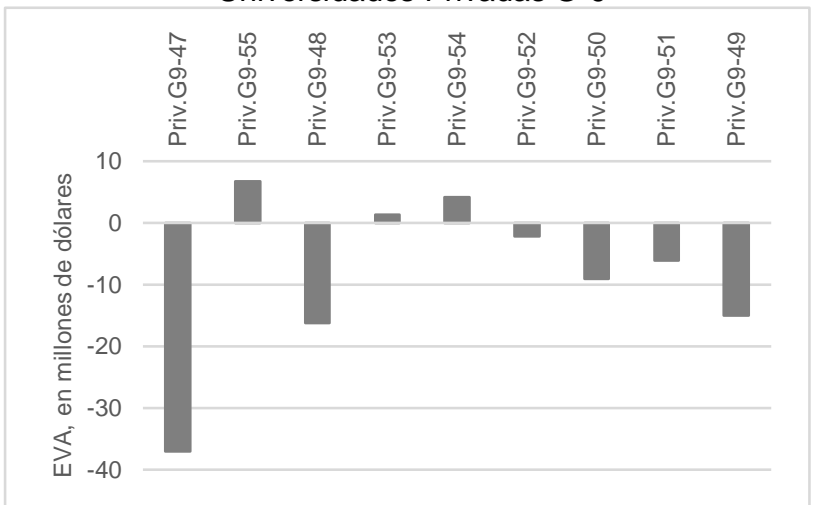

Universidades Privadas B

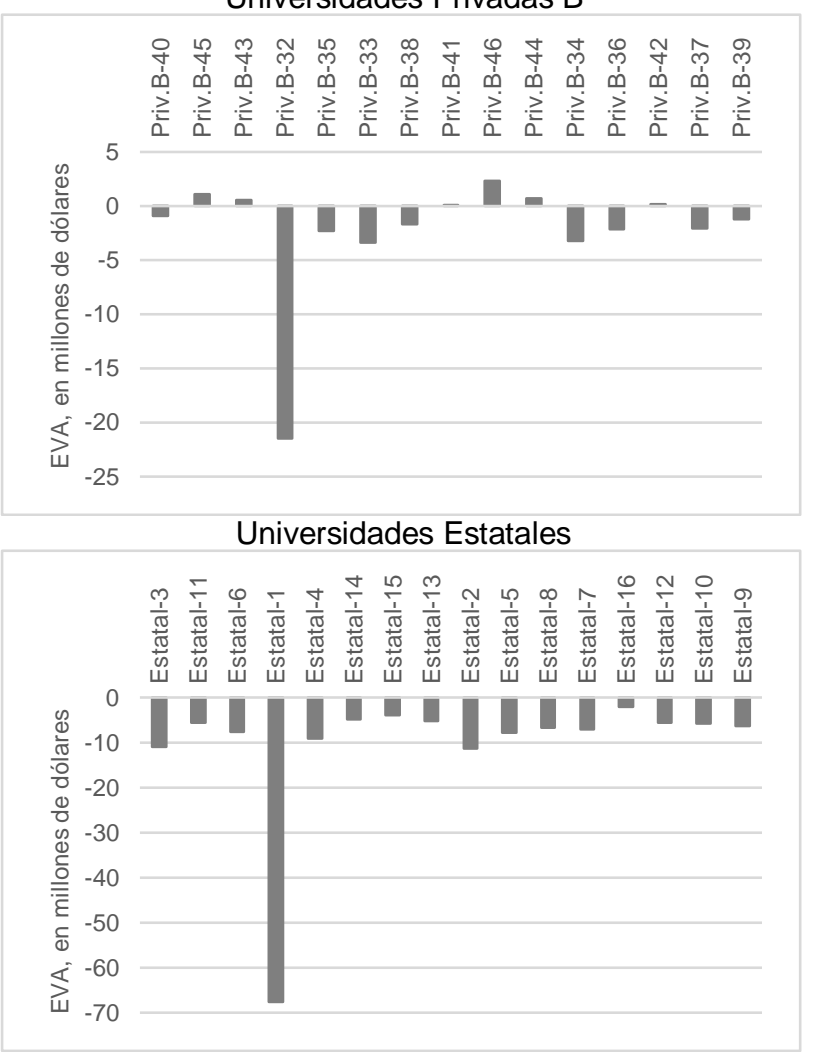

Fig. 1. Estimación de EVA, por universidad y grupo de universidad. Año 2017 (datos de estados financieros auditados 2017 del SIES). 
Tabla 3: Resultados del creación-destrucción de valor EVA a nivel de cada universidad. Año 2017 (datos de estados financieros auditados 2017 del SIES).

\begin{tabular}{|c|c|c|c|c|c|c|}
\hline Universidad & Grupo & Activos & Patrimonio & $\begin{array}{l}\text { Resultado } \\
\text { Operacional }\end{array}$ & $\begin{array}{l}\text { Costo del } \\
\text { Capital }\end{array}$ & EVA \\
\hline U. de Chile & Estatal-1 & 1200,00 & 954,13 & 4,18 & $(71,72)$ & $(67,54)$ \\
\hline U. de Santiago de Chile & Estatal-2 & 284,28 & 219,86 & 5,71 & $(17,06)$ & $(11,34)$ \\
\hline U. Arturo Prat & Estatal-3 & 157,20 & 110,77 & $(1,51)$ & $(9,43)$ & $(10,94)$ \\
\hline U. de La Frontera & Estatal-4 & 173,46 & 144,76 & 1,29 & $(10,41)$ & $(9,12)$ \\
\hline U. de Talca & Estatal-5 & 207,71 & 166,54 & 4,58 & $(12,46)$ & $(7,88)$ \\
\hline U. de Atacama & Estatal-6 & 96,59 & 84,28 & $(1,90)$ & $(5,80)$ & $(7,69)$ \\
\hline U. de Valparaíso & Estatal-7 & 167,54 & 95,64 & 2,93 & $(10,05)$ & $(7,12)$ \\
\hline U. de Tarapacá & Estatal-8 & 174,79 & 159,67 & 3,82 & $(10,49)$ & $(6,66)$ \\
\hline U. Tecnológica Metropolitana & Estatal-9 & 135,08 & 121,79 & 1,80 & $(8,11)$ & $(6,31)$ \\
\hline U. P. A. de Ciencias de la Educación & Estatal-10 & 77,48 & 43,55 & $(1,16)$ & $(4,65)$ & $(5,80)$ \\
\hline U. de Antofagasta & Estatal-11 & 142,47 & 109,90 & 2,92 & $(8,55)$ & $(5,62)$ \\
\hline U. Met. de Ciencias de la Educación & Estatal-12 & 80,39 & 66,94 & $(0,76)$ & $(4,82)$ & $(5,58)$ \\
\hline U. de Magallanes & Estatal-13 & 73,67 & 45,17 & $(0,86)$ & $(4,42)$ & $(5,28)$ \\
\hline U. de La Serena & Estatal-14 & 79,27 & 55,84 & $(0,16)$ & $(4,76)$ & $(4,92)$ \\
\hline U. de Los Lagos & Estatal-15 & 90,43 & 60,73 & 1,57 & $(5,43)$ & $(3,85)$ \\
\hline U. del Bío-Bío & Estatal-16 & 93,44 & 71,89 & 3,59 & $(5,61)$ & $(2,02)$ \\
\hline U. de Los Andes & Priv.A-17 & 456,70 & 214,51 & $(20,14)$ & $(27,40)$ & $(47,54)$ \\
\hline U. Nacional Andrés Bello & Priv.A-18 & 449,14 & 378,17 & 6,84 & $(26,95)$ & $(20,10)$ \\
\hline U. Diego Portales & Priv.A-19 & 199,53 & 78,21 & 5,79 & $(11,97)$ & $(6,19)$ \\
\hline U. Mayor & Priv.A-20 & 264,29 & 76,01 & 10,17 & $(15,86)$ & $(5,69)$ \\
\hline U. Adolfo Ibáñez & Priv.A-21 & 119,39 & 71,40 & 1,57 & $(7,16)$ & $(5,59)$ \\
\hline U. Central de Chile & Priv.A-22 & 129,07 & 88,64 & 2,57 & $(7,74)$ & $(5,17)$ \\
\hline U. Finis Terrae & Priv.A-23 & 81,94 & 33,09 & 1,48 & $(4,92)$ & $(3,44)$ \\
\hline U. Autónoma de Chile & Priv.A-24 & 231,45 & 166,09 & 11,81 & $(13,89)$ & $(2,07)$ \\
\hline U. Católica Cardenal Silva Henríquez & Priv.A-25 & 40,43 & 28,60 & 0,81 & $(2,43)$ & $(1,61)$ \\
\hline U. Alberto Hurtado & Priv.A-26 & 45,19 & 16,78 & 1,23 & $(2,71)$ & $(1,48)$ \\
\hline U. Academia de Humanismo Cristiano & Priv.A-27 & 17,10 & 14,37 & 0,26 & $(1,03)$ & $(0,76)$ \\
\hline U. Bernardo O'Higgins & Priv.A-28 & 43,56 & 27,14 & 1,85 & $(2,61)$ & $(0,76)$ \\
\hline U. del Desarrollo & Priv.A-29 & 164,10 & 47,32 & 9,51 & $(9,85)$ & $(0,33)$ \\
\hline U. de Viña del Mar & Priv.A-30 & 24,67 & 4,61 & 1,33 & $(1,48)$ & $(0,15)$ \\
\hline U. San Sebastián & Priv.A-31 & 225,79 & 88,45 & 17,56 & $(13,55)$ & 4,01 \\
\hline U. de Las Américas & Priv.B-32 & 122,91 & 92,52 & $(14,12)$ & $(7,37)$ & $(21,49)$ \\
\hline U. Gabriela Mistral & Priv.B-33 & 12,11 & 3,17 & $(2,62)$ & $(0,73)$ & $(3,35)$ \\
\hline U. Pedro de Valdivia & Priv.B-34 & 30,59 & 30,59 & $(1,41)$ & $(1,84)$ & $(3,24)$ \\
\hline U. del Pacífico & Priv.B-35 & 14,54 & $(5,16)$ & $(1,40)$ & $(0,87)$ & $(2,28)$ \\
\hline U. Santo Tomás & Priv.B-36 & 96,27 & 53,90 & 3,63 & $(5,78)$ & $(2,14)$ \\
\hline U. UCINF & Priv.B-37 & 1,20 & $(1,92)$ & $(1,99)$ & $(0,07)$ & $(2,06)$ \\
\hline U. Internacional SEK & Priv.B-38 & 31,61 & 19,13 & 0,22 & $(1,90)$ & $(1,67)$ \\
\hline U. UNIACC & Priv.B-39 & 24,68 & 3,56 & 0,27 & $(1,48)$ & $(1,22)$ \\
\hline U. Adventista de Chile & Priv.B-40 & 17,55 & 11,92 & 0,13 & $(1,05)$ & $(0,93)$ \\
\hline U. La República & Priv.B-41 & 10,73 & 4,86 & 0,72 & $(0,64)$ & 0,08 \\
\hline U. Tecnológica de Chile INACAP & Priv.B-42 & 319,08 & 261,00 & 19,34 & $(19,14)$ & 0,19 \\
\hline U. de Aconcagua & Priv.B-43 & 22,23 & 1,87 & 1,89 & $(1,33)$ & 0,55 \\
\hline U. Miguel de Cervantes & Priv.B-44 & 5,57 & 3,14 & 1,07 & $(0,33)$ & 0,74 \\
\hline U. Bolivariana & Priv.B-45 & 8,48 & 3,89 & 1,62 & $(0,51)$ & 1,11 \\
\hline U. Los Leones & Priv.B-46 & 9,94 & 7,11 & 2,91 & $(0,60)$ & 2,31 \\
\hline Pontificia U. Católica de Chile & Priv.G9-47 & 930,68 & 626,12 & 18,74 & $(55,84)$ & $(37,10)$ \\
\hline U. Austral de Chile & Priv.G9-48 & 244,19 & 173,96 & $(1,59)$ & $(14,65)$ & $(16,24)$ \\
\hline U. Técnica Federico Santa María & Priv.G9-49 & 305,29 & 207,93 & 3,28 & $(18,32)$ & $(15,03)$ \\
\hline U. Católica del Norte & Priv.G9-50 & 187,81 & 154,14 & 2,15 & $(11,27)$ & $(9,11)$ \\
\hline U. de Concepción & Priv.G9-51 & 782,57 & 314,67 & 40,90 & $(46,95)$ & $(6,05)$ \\
\hline U. Católica del Maule & Priv.G9-52 & 86,11 & 67,88 & 2,96 & $(5,17)$ & $(2,21)$ \\
\hline U. Católica de la Santísima Concepción & Priv.G9-53 & 97,21 & 54,04 & 7,11 & $(5,83)$ & 1,28 \\
\hline U. Católica de Temuco & Priv.G9-54 & 93,72 & 43,43 & 9,73 & $(5,62)$ & 4,10 \\
\hline Pontificia U. Católica de Valparaíso & Priv.G9-55 & 178,19 & 114,50 & 17,48 & $(10,69)$ & 6,79 \\
\hline
\end{tabular}


Por otro lado, la Tabla 4 muestra aspectos financieros interesantes de los grupos. En primer lugar, se observa que el patrimonio aportado por los dueños de las universidades privadas nuevas ( $\mathrm{A}$ y $\mathrm{B}$ ) es significativo, superando los US $\$ 1.800 .000 .000$ (no incluye deudas con terceros), y logra superar el patrimonio acumulado por las universidades privadas del G-9. También llama la atención que si bien son las universidades estatales las que tienen menores niveles de endeudamiento, son las universidades privadas del grupo B están bastante menos endeudadas que las de más universidades no estatales. Esto sugiere que el desempeño operativo promedio más bajo de estas universidades puede dificultar la obtención de financiamiento, o alternativamente no encuentran justificado obtener más deuda si no observan oportunidades relevantes para crear valor.

Tabla 4. Indicadores contables, año 2017. Cantidades en miles de dólares estadounidenses.

\begin{tabular}{|l|l|l|l|l|}
\hline Grupo & $\begin{array}{l}\text { Activos } \\
\text { Total }\end{array}$ & $\begin{array}{l}\text { Patrimonio } \\
\text { Total }\end{array}$ & $\begin{array}{l}\text { Deuda } \\
\text { Total }\end{array}$ & Leverage \\
\hline Privadas A & $2,492,349$ & $1,333,388$ & $1,158,961$ & $46.5 \%$ \\
\hline Privadas B & 727,481 & 489,592 & 237,889 & $32.7 \%$ \\
\hline Privadas G-9 & $2,905,771$ & $1,756,659$ & $1,149,112$ & $39.5 \%$ \\
\hline Estatales & $3,229,127$ & $2,511,468$ & 717,659 & $22.2 \%$ \\
\hline Total & $9,354,727$ & $6,091,108$ & $3,263,619$ & $34.9 \%$ \\
\hline
\end{tabular}

Determinantes de la creación de valor

Ahora se analiza las variables que parecen explicar mejor la creación de valor por parte de las universidades chilenas. Para esto, varias regresiones lineales de corte transversal son propuestas, utilizando el EVA como una variable explicada. Este tipo de regresiones sobre medidas de desempeño financiera se han utilizado ampliamente en la literatura, tanto para estimaciones predictivas como explicativas (Berger and Humphrey, 1997; Jiang y Lee, 2007).

En primer lugar, considerando el número de estudiantes matriculados (como proxy del tamaño de la universidad), pudiera postularse que las universidades más pequeñas pueden tener peor rendimiento general. Sin embargo, en el modelo 1, la Tabla 5 muestra que no existe una relación estadística entre el tipo de universidad (una variable categórica ordenada) y la creación de valor. Por otro lado, muchas universidades tienen su campus principal en el centro del país (Santiago), aunque también tienen campus más pequeños en el norte y el sur del país. Por localización se entiende aquí a la región donde se encuentra el campus principal de cada universidad. La Tabla muestra que tampoco se encuentra para la localización geográfica de la casa central de las universidades (modelo 2). Por el contrario, los modelos 3 y 4 de la Tabla sugieren que el número de estudiantes (tamaño de la institución) y el número de años de acreditación (como proxy de la calidad) parecen jugar un papel explicativo, a niveles estándar de significancia estadística. Finalmente, el modelo 5 incluye todas las variables conjuntamente, y se observa que en este caso es solo el tamaño de la universidad lo que mejor ayuda a explicar su desempeño. El signo negativo del coeficiente muestra que la relación es inversa y, en consecuencia, cuanto mayor es el tamaño mayor es la destrucción del valor. El hecho de que los años de acreditación pierda valor explicativo cuando se formula junto con la inscripción, parece indicar que gran parte de la información que parecía contener ya está incluida en la variable "matrícula".

Tabla 5. Especificaciones explicativas alternativas de creación de valor en universidades chilenas. Datos año 2017 (miles de US\$). ${ }^{*} \mathrm{p}<0.10,{ }^{* *} \mathrm{p}<0.05,{ }^{* * *} \mathrm{p}<0.01$ (datos de estados financieros auditados 2017 del SIES).

\begin{tabular}{|l|l|l|l|l|l|}
\hline & (modelo 1) & (modelo 2) & (modelo 3) & (modelo 4) & (modelo 5) \\
\hline tipo_universidad & -1800.0 & & & & -1400.0 \\
\hline & $(-1.28)$ & & & & $(-1.10)$ \\
\hline & & & & & \\
\hline Iocalizacion & & 1751.1 & & & 3031.0 \\
\hline & & $(0.54)$ & & & $(1.07)$ \\
\hline & & & & & \\
\hline matricula_2017 & & & $-0.538^{\star * *}$ & & $-0.446^{\star *}$ \\
\hline & & & $(-3.69)$ & & $(-2.64)$ \\
\hline & & & & & \\
\hline anos_acreditacion & & & & $-2500.0^{* * *}$ & -1200.0 \\
\hline & & & & $(-3.31)$ & $(-1.37)$ \\
\hline & & & & & \\
\hline cons & -2300.0 & -10000.0 & 382.9 & 2443.3 & 974.1 \\
\hline & $(-0.60)$ & $(-1.52)$ & $(0.16)$ & $(0.77)$ & $(0.14)$ \\
\hline$N$ & 55 & 55 & 55 & 55 & 55 \\
\hline
\end{tabular}




\section{CONCLUSIONES}

A partir de los resultados obtenidos, se pueden extraer las siguientes conclusiones: 1) Los cuatro grupos de universidades chilenas destruyeron valor en el año 2017, es decir generaron un EBIT medio más bajo que el coste de capital; 2) Las universidades privadas del Grupo A (los que tienen la más alta calidad académica) tienen características similares a las universidades privadas del G-9 en cuanto al grado de creación de valor, y a su estructura financiera. Sin embargo, si se considera que prácticamente no reciben fondos estatales, se puede concluir que estos son el grupo más eficiente en el uso de recursos; 3) Cuatro universidades se destacan por presentar resultados especialmente negativos en comparación con las otras universidades de su grupo: U. de Los Andes, U. de Las Américas, Pontificia U. Católica de Chile, y U. de Chile; 4) Todas las universidades estatales presentan EVA negativos, contrario a los otros grupos, en que se tienen resultados mixtos; 5) El mejor predictor de desempeño fue el tamaño de las universidades, aunque con un signo inverso, es decir, son las universidades más grandes las que estadísticamente enfrentan mayor destrucción de valor; y 6) falta bastante camino para recorrer en la construcción de estimadores consensuados de creación de valor para organizaciones sin fines de lucro, siendo este estudio una contribución en esa dirección.

\section{REFERENCIAS}

Aguillo, I., Bar-Ilan, J. y otros dos autores, Comparing university rankings, Scientometrics, 85, 243-256 (2010)

Al Mamun, A., Entebang, H. y Mansor, S. A., EVA as Superior Performance Measurement Tool, Modern Economy, 3, 310 (2012)

Baran, P. Werte, Europäische Enzyklopädie zu Philosophie und Wissenschaften, In: H. J. Sandkühler (Ed.), Vol. 4, pp. 805-815. Hamburg: Felix Meiner Verlag, 806 (1991)

Berger, A.N. y Humphrey, D.B., Efficiency of financial institutions: international survey and directions for future research, European Journal of Operational Research, Vol 98, pp.175-212 (1997)

Bornmann, L., De Moya Anegón, F., Leydesdorff, L., The new Excellence Indicator in the World Report of the SClmago Institutions Rankings 2011, Journal of Informetrics, 6 (2), pp. 333-335. dx.doi.org/10.1016/j.joi.2011.11.006 (2012)

Charnes A., Cooper, W. y Rhodes, E., Measuring the Efficiency of Decision Making Units, European Journal of Operational Research 2 (1978) 429-444 (1978)

CGR, Contraloría General de la República de Chile, Financiamiento Fiscal a la Educación Superior, Informes anuales. www.contraloria.cl (2017)

Cruz-Coke, R., The evolution of Chilean universities from 1981 to 2004. Rev Méd Chile 2004; 132: 1543-1549 (2004)

Daraio, C., Bonaccorsi, A., Simar, L., Efficiency and economies of scale and specialization in European universities: a directional distance approach. Journal of Informetrics 9 (3), 430-448 (2015)

Díaz, W., Aproximación al Valor Económico Agregado (Economic Value Added, EVA) en organizaciones públicas, Universidad y Empresa, 8(16), 215-241 (2009)

Grant, J. L., Foundations of Economic Value Added. 2nd Edition Hardcover: 324 pages. Wiley (2002)

Hasani, S. M., y Fathi, Z., Relationship the Economic Value Added (EVA) with Stock Market Value (MV) and Profitability Ratios, Interdisciplinary Journal of Contemporary Research in Business, 4(3), 406-415 (2012)

Jiang, X. y Lee, B. S., Stock returns, dividend yield, and book-to-market ratio. Journal of Banking \& Finance, 31, 455-475 (2007)

Meynhardt, T., Public Value Inside: What is Public Value Creation?, Intl Journal of Public Administration, 32: 192-219. DOI: 10.1080/01900690902732632 (2009)

Moore, M. H., Creating Public Value. Strategic Management in Government. Cambridge, MA: Harvard University Press (1995)

Porter, S.R. y Toutkoushian, R.K., Institutional research productivity and the connection to average student quality and overall reputation. Econ. Educ. Rev. 25 (6), 605-617 (2006)

Porter M.E. y Kramer M.R., Creating Shared Value. In: Lenssen G., Smith N. (eds) Managing Sustainable Business. Springer, Dordrecht (2019)

Ramírez, P.E. y Alfaro, J.L., Evaluation of the efficiency of the universities belonging to the council of rectors of Chilean universities: results of a data envelopment analysis. Formacion Universitaria 6 (3), 31-38 (2013)

Rescher, N., Introduction to Value Theory. Washington, DC: University Press of America (1969/1982)

Rompho, N., Application of the Economic Value Added (EVA) Protocol in a University Setting as a Capital Budgeting Tool, Journal of Financial Reporting and Accounting, Vol. 7 Iss: 2, pp.1 - 17 (2009)

Shamohammadi, M. y Dong-hyun O., Measuring the efficiency changes of private universities of Korea: A two stage network data envelopment analysis, Technological Forecasting \& Social Change, 148 (2019) 
Sharma, A. K. y Kumar, S., Economic Value Added (EVA) - Literature Review and Relevant Issues, International Journal of Economics and Finance, 2(2) (2010)

Stern, J., Stewart, G. y Chew, D., The EVA financial management system, Journal of Applied Corporate Finance, 8(2), 3246 (1995). doi: 10.1111/j.1745-6622.1995.tb00285.x

Stern, J. y Shierly, J., The EVA Challenge: Implementing Value-Added Change in an Organization. New York: John Wiley \& Sons, Inc (2001)

Tang, S., Academic quality characteristics and satisfaction: An empirical survey among the students of two Malaysian private universities, Academic Research International, 2 (2012)

UNESCO, The Role of Higher Education in Society: Quality and Pertinence, New Papers on Higher Education Meeting Documents. 2nd UNESCO- Non-Governmental organizations Collective Consultation on Higher Education Paris, 8-11 April (1991)

Wilkinson, R., Yussof, I., Public and private provision of higher education in Malaysia: A comparative analysis, Higher Education, 50, 361-366 (2005)

Zuniga-Jara, S., Barraza, J. y otros dos autores, Midiendo la Creación de Valor en una Universidad, doi: 10.4067/S071850062018000300087, Formación Universitaria, 11(3), 87-96 (2018) 
